\title{
RELATOS DE PESQUISA
O ESTÁGIO DE DESENVOLVIMENTO DA GESTÃO DO
CONHECIMENTO NOS INSTITUTOS FEDERAIS DE
EDUCAÇÃO, CIÊNCIA E TECNOLOGIA
}

José Nivaldo Balbino

Mestre em Ciência, Gestão e Tecnologia Informação pela Universidade Federal do Paraná, Brasil. Administrador do Instituto Federal de Educação, Ciência e Tecnologia do Paraná, Brasil.

E-mail: jnbalbino@gmail.com

Helena de Fátima Nunes

Doutora em Engenharia de Produção pela Universidade Federal de Santa Catarina, Brasil. Professora da Universidade Federal do Paraná, Brasil.

E-mail: helenanuness@gmail.com

Fernanda Cristina Barbosa Pereira Queiroz

Doutora em Engenharia de Produção pela Universidade Federal de Santa Catarina, Brasil. Professora da Universidade Federal do Rio Grande Norte, Brasil.

E-mail: fbp@ufrnet.br

\begin{abstract}
Resumo
O presente artigo apresenta uma categorização conceitual da Gestão do Conhecimento e adota o Modelo de Maturidade de Gestão do Conhecimento para a Administração Pública, desenvolvido por Fábio Ferreira Batista, com a finalidade de analisar o estágio de desenvolvimento da gestão do conhecimento no âmbito dos Institutos Federais de Educação, Ciência e Tecnologia (IFs). Trata-se de uma pesquisa quantitativa e qualitativa, exploratória e descritiva, cuja coleta de dados pauta-se em um questionário adaptado, utilizado com o fim de mensurar o desenvolvimento do estágio de maturidade da gestão do conhecimento nestas Instituições de Ensino. Os resultados obtidos apontam o nível atual de desenvolvimento de cada uma das áreas da gestão do conhecimento em relação às instituições que apresentaram suas devolutivas. Os dados coletados permitiram individualizar a intensidade das iniciativas da gestão do conhecimento perpetradas internamente pelos IFs. Conclui que o ambiente organizacional estudado encontra-se no nível inicial de maturidade, ou seja, a adoção de práticas voltadas à implementação da gestão do conhecimento nestas Instituições de Ensino atua de forma embrionária. Observou-se, ainda, que as instituições de ensino mais bem niveladas de acordo com o critério adotado possuem suas atenções voltadas aos critérios de tecnologia e aprendizagem e inovação.
\end{abstract}

Palavras-chave: Gestão do Conhecimento. Modelo de Maturidade. Administração Pública.

\section{THE MANAGEMENT OF THE DEVELOPMENT STAGE OF KNOWLEDGE IN THE INSTITUTES OF FEDERAL EDUCATION, SCIENCE AND TECHNOLOGY}

\section{ABSTRACT}

This article presents a conceptual categorization of knowledge management and adopts the Model of Knowledge Management Maturity for Public Administration, developed by Fábio Ferreira Batista with the purpose of examining the stage of knowledge management stage in the context of the Federal

Perspectivas em Gestão \& Conhecimento, João Pessoa, v. 6, n. 2, p. 80-98, jul./dez. 2016. http://periodicos.ufpb.br/ojs2/index.php/pgc. ISSN: 2236-417X. Publicação sob Licença (cc) EY-NC-ND 
Institutes of Education, Science and Technology. This elements design a better research on the maturity of institutional knowledge management. Therefore, this study is based on a quantitative and qualitative, exploratory and descriptive research. Its data collection is guided in an adapted questionnaire used in order to measure the stage of maturity development of knowledge management in these institutions. The obtained results indicate the current level of development of each area of knowledge management in relation to institutions that presented their devolution. The collected data allows individualizing the intensity of knowledge management initiatives carried out internally by the Federal Institutes of Education, Science and Technology. It concluded that the studied organizational environment is the initial maturity level, which can be explained by the adoption of practices aimed at implementation of knowledge management in these education institutions acts in an embryonic form. It was also observed that most educational institutions well leveled according to the criteria adopted are focused on the criteria for technology and learning and innovation.

Keywords: Knowledge Management. Maturity Model. Public Administration.

\section{INTRODUÇÃO}

A gestão do conhecimento é objeto de pesquisa em âmbito nacional e internacional, com foco, na maioria dos estudos, em organizações privadas. No setor público, as pesquisas centram-se em áreas específicas, como por exemplo, no papel da tecnologia ou serviços de governo eletrônico (LING, 2002; JAIN, 2009); ou polícia (DALE, 2001; LUEN E AL- HAWAMDEH, 2001) e de saúde (VAN BEVEREN, 2003). A pesquisa de Alatawi, Dwivedi e Williams (2013) sobre gestão do conhecimento no setor público, em 51 países em desenvolvimento e 20 países árabes, revelou que em ambas as regiões, a maioria dos estudos são conduzidos em setores específicos como saúde, agricultura, telecomunicações e defesa.

Wiig (2002) em seu estudo sobre a gestão do conhecimento na administração pública investigou como esta pode desempenhar um papel importante na administração pública, particularmente em quatro áreas principais: (WIIG, 2002): (1) melhorar a tomada de decisões no âmbito dos serviços públicos; (2) ajudar o público em participar, efetivamente, nas tomadas de decisões; (3) construir capacidades de capital intelectual da sociedade competitivos; (4) desenvolver uma força de trabalho de gestão do conhecimento. Tal abordagem pode permitir "a sociedade prosperar e aumentar a sua viabilidade, fazendo seu povo e as instituições trabalharem mais espertos" (WIIG, 2002, p. 238), e, além disso, dar benefícios e aumentar a qualidade de vida dos cidadãos.

No Brasil, existem experiências de implementação da gestão do conhecimento em órgãos públicos nas esferas federal, estadual e municipal e poderes executivo, legislativo e judiciário. Alguns estudos ilustram essas ações como: Carvalho (2008) que apresentou estratégias de implementação no Serviço Federal de Processamento de Dados (Serpro); Goulart (2008) que mapeou os conhecimentos necessários para realização dos objetivos estratégicos na Caixa Econômica Federal (CEF); Silva (2008) que relatou a experiência na Prefeitura Municipal de Curitiba (PMC) e teve como objetivo demonstrar a relação direta com as comunidades de prática e a valorização dos seus servidores, por meio de compartilhamento do conhecimento e na criação de novas ideias; Angeloni e Mussi (2008) que descreveram como ocorre o compartilhamento do conhecimento em um projeto de tecnologia da informação referente à implementação de um sistema ERP (Enterprise Resource Planning) na Universidade de Santa Catarina (Unisul); Oliveira e Torres (2008) que abordaram sobre a inovação e pesquisa em desenvolvimento no Setor Elétrico na Chesf (Companhia Hidro Elétrica do São Francisco); Fiates (2008) que relatou sobre o contexto da Cultura Organizacional da Companhia Vale do Rio Doce (CVDR) visando a valorização das pessoas e do conhecimento na organização.

Perspectivas em Gestão \& Conhecimento, João Pessoa, v. 6, n. 2, p. 80-98, jul./dez. 2016 
Abdullah e Date (2009, p.4) sugerem quatro características a serem observadas pelo setor no desenvolvimento de ações de gestão do conhecimento (a) o conhecimento é um recurso inimitável do governo; governo efetivo repousa sobre efetiva aquisição e disseminação de conhecimento; (b) o governo é uma empresa distribuída, portanto, requisitos de conhecimento semelhantes estão distribuídos nos estados e governos locais; (c) transferências frequentes de trabalhadores do conhecimento em todos os departamentos governamentais causam problemas de "fuga de conhecimento"; e (d) a necessidade de "governos de antecipação "que aprendem com as experiências do passado, entendem o cenário presente, antecipam ameaças e oportunidades futuras."

Para Edge (2005) a gestão do conhecimento tem o potencial de influenciar e melhorar o setor público nos processos de renovação (Fullan, 2001; McAdam e Reid, 2001). Conforme McAdam e Reid (2001, p.317) acredita-se que a gestão do conhecimento é "particularmente importante no setor público como pessoal e há muito tempo foi identificado como o depositário de conhecimentos chaves".

Em contextos onde a informação e o conhecimento são essenciais para fortalecer as competências organizacionais e consolidar vantagens competitivas sustentáveis, a Gestão do Conhecimento deve ser compreendida como o conjunto de atividades que almejam a promoção do conhecimento organizacional, fornecendo as melhores informações e conhecimentos disponíveis aos seus colaboradores com o intuito de atingir os objetivos e a maximização da competitividade (ALVARENGA NETO, 2008).

A Gestão do Conhecimento pode ser compreendida "como um conjunto de processos que visam à criação, armazenamento, disseminação e utilização do conhecimento, alinhados com os objetivos do negócio, considerando fontes de conhecimento internas e externas à organização" (OLIVEIRA et al., 2011, p.12).

Em contraponto, as demandas relacionadas à identificação do progresso na implementação e na análise dos resultados alcançados por meio da gestão do conhecimento ainda são complexas para a maioria das organizações (OLIVEIRA et al., 2011), em especial as de caráter público. Observou-se, a partir do estudo exploratório, que a avaliação do grau de maturidade da gestão do conhecimento em organizações públicas é rara e inexistente nos IFs, objeto de análise empírica.

A escolha dos IFs para realização da pesquisa se deu pela importância da organização no contexto educacional brasileiro e pelos resultados que a sociedade espera em termos de qualificação técnica de qualidade aos cidadãos. As ações governamentais, nesta perspectiva, têm por finalidade a busca pela excelência nas práticas desenvolvidas pelo Estado destinadas às políticas institucionais e programas sociais de qualificação profissional, inserindo os participantes no processo de produção e conferindo-lhes inclusão social.

Logo, os IFs têm por finalidade atender às necessidades sociais, ofertando educação profissional e tecnológica para prover às políticas públicas de educação. Isto confere igualdade à diversidade social existente, seja de cunho econômico, social, cultural, dentre outros. Tais práticas resultam na articulação com outras políticas públicas sociais, tais como trabalho e renda, desenvolvimento setorial, etc., que impactam diretamente o contexto em que a sociedade está inserida (PEREIRA, 2008).

As instituições de ensino tecnológicas têm duplo papel. São educadoras e formadoras dos trabalhadores. E, neste sentido, exige-se um constante repensar das suas práticas de gestão, as quais devem possibilitar a implementação de ações modernizadoras clamadas diretamente pela sociedade que objetiva o rompimento de práticas antagônicas, morosas, não-efetivas e ineficientes, especialmente porque o espaço social contempla não só as organizações públicas, como também as privadas, tornando-se essencial a adoção de práticas oriundas da gestão do conhecimento, com vistas a transcender aos limites do desempenho 
organizacional conferindo melhor qualidade aos serviços públicos prestados (MARICATO, et al., 2013).

Assim, o estudo apresentado volta-se a compreender os estágios de desenvolvimento da gestão do conhecimento na administração pública, mediante uma avaliação comparativa dos IFs, por meio do modelo de gestão pública do conhecimento para a administração pública brasileira (BATISTA, 2012), com vistas a determinar o nível de maturidade da gestão do conhecimento neste setor da administração pública. Esta investigação é necessária frente à importância conferida ao conhecimento para o desenvolvimento institucional, notadamente pelo aspecto da transição ocorrida nos ambientes social e econômico na Sociedade da Informação.

\section{GESTÃO DO CONHECIMENTO NA ADMINISTRAÇÃO PÚBLICA}

A Gestão do Conhecimento tornou-se disciplina autônoma a partir de 1991, por intermédio da contribuição das seguintes áreas do saber: a Administração, o Sistema de Informações, a Biblioteconomia e a Ciência da Informação. As disciplinas de Comunicação, Ciência da Computação, Saúde Pública e Políticas Públicas, recentemente, concorreram para sua formatação nos moldes reconhecidos neste século (RAMANIGOPAL, 2012).

Ramanigopal (2012) enfatiza que a Gestão do Conhecimento compõe um conjunto de estratégias e práticas que permitem uma reestruturação organizacional, capaz de reconhecer pontos fracos; inovar, e; reorganizar funções voltadas à otimização organizacional. Essas ações permitem a implementação de novidades e a ampliação de boas experiências que compreenderão o conhecimento incorporado por indivíduos tendenciosos à melhoria gerada no ambiente organizacional, por intermédio de processos ou de práticas administrativas.

A relevância da Gestão do Conhecimento lhe confere presença ativa na maioria das organizações, de cunho público ou privado, ao desempenhar funções imprescindíveis ao desenvolvimento da gestão nas mais variadas áreas organizacionais (RAMANIGOPAL, 2012).

As organizações, em seu ambiente interno e nos relacionamentos externos, necessitam administrar as informações que lhe são afetas como forma de adaptação, inclusive para a busca de prosperidade num espaço cada vez mais dinâmico (CHOO, 2006).

$\mathrm{Na}$ administração pública, a gestão do conhecimento volta-se a gerir informações destinadas à manutenção, pelo Estado, das atividades consideradas essenciais e indispensáveis ao convívio numa sociedade democrática de direito (SCHLESINGER et al., 2008). Assim, as transformações econômicas e sociais, embora atinjam os setores públicos e privados, são ponderadas com mais cautela na administração pública. Esta, na maioria das vezes, atua na retaguarda das mudanças sociais, especialmente porque o agir estatal reflete diretamente no contexto que o corporifica.

A prestação dos serviços públicos tem em sua essência uma dualidade intrínseca ao ambiente estatal. Para tanto, as atividades desenvolvidas pelo Estado voltam-se à satisfação do interesse público e, ao mesmo tempo, à obtenção de resultados eficazes, de acordo com a sistemática normativa aplicável ao contexto social. Portanto, a mutação presente neste cenário atinge diretamente a administração e os administrados. E, estes últimos, estão cada vez mais conscientes de seus direitos, ao ampliarem o grau de exigência em relação às atuações prestacionais do Estado (SCHLESINGER et al., 2008).

Os agentes que compõem a Administração Pública atuam em nome da sociedade, ao expressar suas preferências e valores. Todavia, os contextos social e administrativo encontram-se em constante mutação pela própria dinâmica que permeia o convívio entre os indivíduos. Isso permite uma retroalimentação sincrética de alteração tanto do contexto social quanto administrativo e, consequentemente, gera relevantes alterações das ações implementadas em nome do Estado (BOURGON, 2007). 
As tentativas de reciclagem da Administração Pública para acompanhar o contexto social podem ser vistas por meio dos modelos administrativos aplicados até então, partindo-se do modelo clássico, com enfoque no controle e desenho organizacional, para o modelo neoburocrático, sedimentado no processo de decisão racional. Além destes modelos, também foi adotado o modelo institucional, fortemente difundido entre os anos de 1950 e 1960, com ênfase nas ciências do comportamento. Por fim, adotou-se o modelo público de escolha, pautado na economia política (DENHARDT; DENHARDT, 2000).

Seguindo este viés, a Gestão do Conhecimento é hábil a aumentar a capacidade do conhecimento dos agentes públicos no âmbito da administração pública. A Gestão do Conhecimento aplica-se, portanto, de maneira coletiva, sistemática e integrada, com a finalidade de inovar e compartilhar o conhecimento para atingir os resultados pretendidos pelo Estado (BATISTA, 2012).

Ao discorrer a respeito das características evidenciadas para o desenvolvimento gerencial das novas ações estatais, Bourgon (2007) afirma que referidas mudanças podem ser observadas nas práticas administrativas de diversos Estados, com ramificações profundas em relação ao papel do governo. Estas práticas, no entender do autor citado, exigem a inclusão de uma nova teoria da administração pública.

A Gestão do Conhecimento se apresenta como resposta adequada a esta nova teoria da administração pública. É desenvolvida a partir dos ensinamentos oferecidos por Adbullah e Date (2009), segundo os quais as a gestão do conhecimento no setor público deve contemplar as seguintes características:

i) o conhecimento é compreendido como recurso peculiar de cada governo que terá por função não só adquirir como disseminá-lo em seu meio;

ii) o governo é constituído enquanto uma organização com competências e atribuições divididas em esferas, cujo conhecimento deve transitar entre os diversos meios sem que haja pontos de contenção em razão da necessidade dos interesses locais, estaduais e nacionais.

iii) a rotatividade de servidores, notadamente no âmbito do Poder Executivo gera problemas atinentes à fuga do conhecimento em razão do corte da linearidade necessária para sua transmissão e disseminação;

iv) o aprendizado governamental com as experiências ocorridas no passado permite melhor interpretar o cenário atual, bem como antecipar ameaças e oportunidades futuras.

Essa conjuntura indica uma maior habilidade da Gestão do Conhecimento em influenciar e melhorar o setor público no seu processo de renovação, especialmente em setores marcados pela rotatividade dos profissionais. Em tais ambientes, torna-se imprescindível a adoção de práticas tendentes a capturar o conhecimento dos servidores, propensos a valorizar a construção do conhecimento pela confluência dos elementos científicos e sociais envolvidos (MCADAM; REID, 2001).

Segundo Wiig (2002) a Gestão do Conhecimento é fundamental para conferir maior assertividade nas ações de tomada de decisão praticadas no setor público; ajudar a coletividade a participar de forma efetiva destas tomadas de decisões; construir capacidades competitivas no desenvolvimento do capital intelectual da sociedade, e; desenvolver uma força de trabalho de gestão do conhecimento no setor público.

Portanto, a relevância da Gestão do Conhecimento atrela-se à melhoria das práticas administrativas internas ao setor público, bem como ao contato direto pelo Estado com a sociedade e os demais entes privados. Isto evidencia a distinção subjacente ao ambiente 
organizacional e aos processos implementados para realização das metas e dos objetivos vinculados à Gestão do Conhecimento pelo Estado.

Acredita-se que o processo de gestão do conhecimento no setor público, em relação à identificação, à criação, ao compartilhamento, ao armazenamento e à aplicação do conhecimento, está ligado à constância da aprendizagem da inovação (BATISTA, 2012). As práticas inovadoras são direcionadas para aumentar a eficiência dos serviços públicos ofertados à população, os quais podem ser avaliados por meio de modelos de maturidade de gestão do conhecimento.

\section{MATURIDADE E MODELOS DE MATURIDADE DE GESTÃO DO CONHECIMENTO}

Para atingir a maturidade, a implantação da Gestão do Conhecimento deve fornecer aos participantes desse processo condições favoráveis ao desenvolvimento de suas aptidões. Esta prática permite alterar a visão de mundo dos participantes, com o objetivo de aumentar os benefícios àqueles que se adaptam a esta prática de gestão, nos planos individual ou organizacional, em relação aos profissionais presentes neste ambiente corporativo. (BARRADAS; CAMPOS FILHO, 2010).

Os modelos de maturidade avaliam as áreas de conhecimento de acordo com os objetivos institucionais propostos. O nível de maturidade equivale ao progresso da orientação estratégica traçada e a sua respectiva implementação nos processos administrativos, passíveis de projeção e análise de resultados (GONÇALO; JUNGES; BORGES, 2010).

A confluência dos modelos de controle de qualidade, de maturidade e de desenvolvimento de software simbioticamente influencia e fortalece os modelos de maturidade em Gestão do Conhecimento. Esta tríade permite realizar uma diagnose dos elementos encontrados, conferindo à organização a capacidade de detectar as virtudes e fraquezas dos recursos de conhecimentos disponíveis. Isso permite a reestruturação dos planos organizacionais e a atualização dos programas de ações a serem desempenhados no ambiente organizacional (FRESNEDA, 2009).

$O$ desenvolvimento e a implantação de qualquer espécie de planejamento voltado às ações de Gestão do Conhecimento dependem da cognição prévia da vivência organizacional. Essa prática permite identificar as necessidades organizacionais e adequá-las ao modelo de maturidade mais apropriado à satisfação dos resultados pretendidos.

A elaboração de mecanismos de mensuração de resultados pautados em métricas, o gerenciamento e o estímulo às práticas de Gestão de Conhecimento são fundamentais à avaliação, ao controle a ao aperfeiçoamento do desenvolvimento organizacional, bem como à implementação de estratégias corporativas (GONÇALO; JUNGES; BORGES, 2010).

Todas estas ações, voltadas à mensuração do conhecimento, são indispensáveis para a organização verificar se atingiu os resultados pretendidos. A métrica constitui recurso apto a aprimorar o desempenho organizacional por permitir compará-lo, além de possibilitar a análise do desempenho dos processos, de equipes e até mesmo de indivíduos de um dado setor.

A dinâmica organizacional, nestes moldes, requer uma Gestão do Conhecimento eficaz para respaldar a tomada de decisão e a execução de atividades voltadas à sobrevivência e a prosperidade organizacional (AHMED; LIM; ZAIRI, 1999).

Assim, a avaliação dos modelos de maturidade vincula-se à compreensão da subjetividade que cerca o ambiente organizacional. Isso torna o processo em referência mais dificultoso em comparação, a título de exemplo, com a realização de levantamento de indicadores por intermédio de um sistema de informação (GONÇALO; JUNGES; BORGES, 2010).

Perspectivas em Gestão \& Conhecimento, João Pessoa, v. 6, n. 2, p. 80-98, jul./dez. 2016 
Sobre o tema, Oliveira et al., (2011) formularam um quadro destinado a compreender a complexidade que permeia os modelos de maturidade de gestão do conhecimento ao analisá-los comparativamente, conforme reproduzido, com acréscimos a seguir:

Quadro 1 - Análise comparativa dos modelos de maturidade de GC

\begin{tabular}{|c|c|c|c|c|c|c|c|c|c|c|c|c|c|c|}
\hline Fatores & 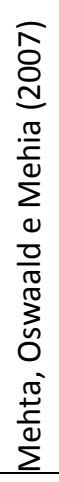 & 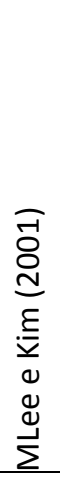 & 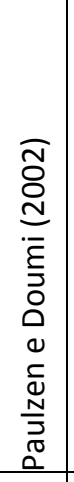 & 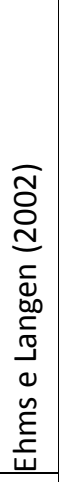 & 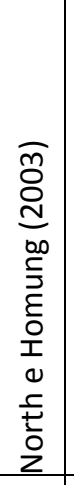 & 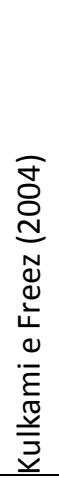 & 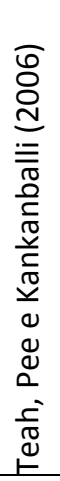 & 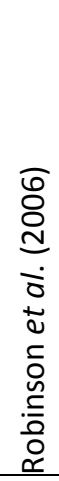 & 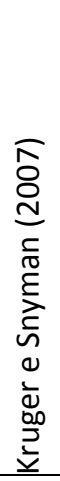 & 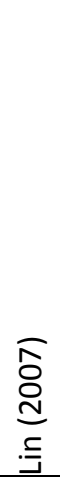 & 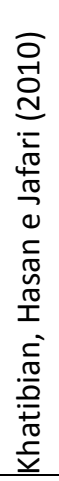 & 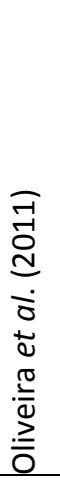 & 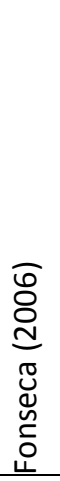 & 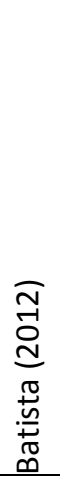 \\
\hline Clientes & & $x$ & $x$ & $x$ & & & & & $x$ & $x$ & & $x$ & & \\
\hline Fornecedores & & $x$ & & $x$ & & & & & $x$ & & & $x$ & & \\
\hline Parceiros & & $x$ & & $x$ & & & & & $x$ & & & $x$ & & \\
\hline Competidores & & $x$ & & & & & & & $x$ & & & $x$ & & \\
\hline Legislação & & & & & & & & & & & & $x$ & $\mathrm{x}$ & $x$ \\
\hline Cultura & $x$ & & & $x$ & & $x$ & $x$ & $x$ & $x$ & $x$ & $x$ & $x$ & $x$ & $x$ \\
\hline Estrutura & & $x$ & & $x$ & & & & & & & $x$ & $x$ & $x$ & $x$ \\
\hline $\begin{array}{l}\text { Suporte da alta } \\
\text { administração }\end{array}$ & & & & $x$ & & $\mathrm{x}$ & & & & $x$ & $x$ & $\mathrm{x}$ & $x$ & $x$ \\
\hline $\begin{array}{l}\text { Alinhamento com os } \\
\text { objetivos de negócio }\end{array}$ & $x$ & & & $x$ & & & $x$ & $x$ & $x$ & & $x$ & $x$ & $x$ & $x$ \\
\hline Objetivos da GC & $x$ & $x$ & & $x$ & & $x$ & & $x$ & $x$ & $x$ & $x$ & $x$ & $x$ & $x$ \\
\hline Benefícios & & & & & & & $x$ & $x$ & $x$ & $x$ & & $x$ & $x$ & $x$ \\
\hline Conhecimento tácito & $x$ & & & & & $x$ & & & $x$ & & & $x$ & $x$ & $x$ \\
\hline Conhecimento explícito & & & & & & $x$ & & $x$ & $x$ & & & $x$ & $x$ & $x$ \\
\hline Conhecimento crítico & & & & & & & & & $x$ & & $x$ & $x$ & $x$ & $x$ \\
\hline Tecnologia & $x$ & & $x$ & $x$ & $x$ & $x$ & $x$ & & $x$ & $x$ & $x$ & $x$ & $x$ & $x$ \\
\hline Líder de GC & & & & & & & $x$ & $x$ & & $x$ & & $x$ & $x$ & $x$ \\
\hline Sistema de recompensa & $x$ & & $x$ & & & $x$ & $x$ & $x$ & & $x$ & $x$ & $x$ & $x$ & $x$ \\
\hline Tempo & & & & & & & & & & & & $x$ & & \\
\hline Treinamento & $x$ & & & & & $x$ & $x$ & & & $x$ & $x$ & $x$ & $x$ & $x$ \\
\hline Fases do processo & $x$ & & $x$ & $x$ & $x$ & $x$ & $x$ & $x$ & $x$ & $x$ & $x$ & $x$ & $x$ & $x$ \\
\hline Comunicação & & $x$ & & $x$ & & $x$ & & $x$ & & $x$ & $x$ & $x$ & $x$ & $x$ \\
\hline Orçamento & & $x$ & & $x$ & & & $x$ & $x$ & & $x$ & & $x$ & $x$ & $x$ \\
\hline Setor Público & & & & & & & & & & & & & $x$ & $x$ \\
\hline
\end{tabular}

Fonte: Oliveira et al. (2011) ampliado pelos Autores (2015) 
A ampliação realizada no Quadro 1 consiste na inclusão da análise comparativa dos modelos de maturidade de Gestão do Conhecimento desenvolvidos por Oliveira et al. (2011), Fonseca (2006) e Batista (2012).

Conforme explicitado neste Quadro 1, uma investigação pormenorizada dos modelos de maturidade pode ser realizada com a adoção de diversos elementos ou pelo enfoque de perspectivas distintas, onde cada qual terá por função constatar a evolução da gestão do conhecimento aplicada ao caso concreto. Todavia, para o âmbito do setor público, as informações do referido Quadro 1 evidenciam que apenas os modelos desenvolvidos por Fonseca (2006) e Batista (2012) são aplicáveis.

O Modelo Organizational Knowledge Assessment (OKA) decorre de um processo iterativo triangular entre Pessoa-Processo-Sistema, indispensável à análise de progresso que poderá se estabelecer em quaisquer destes campos, com a função de descrever a natureza da Gestão do Conhecimento no âmbito interno de uma organização (FONSECA, 2006).

A coleta de dados, necessários à avaliação do conhecimento pertencente a uma dada organização, exige a adoção de uma abordagem qualitativa. Porém, existe pouca estatística padrão destinada a esta finalidade e ainda menos organizações com acesso a estes meios para coletar os dados disponíveis (FONSECA, 2006).

O Método OKA pauta-se em dados subjetivos inerentes à organização analisada e sua aplicabilidade relaciona-se ao fácil acesso aos dados a serem coletados. A coleta de dados e a respectiva avaliação do conhecimento da organização investigada demandam habilidade para o manejo eficiente das informações e dos conhecimentos disponíveis. Isso exige do analista a utilização de variadas competências, como a intuição não só sobre as pessoas, mas também em relação aos processos e ao próprio sistema, tudo com a finalidade maior de agregar valor à organização (FONSECA, 2006).

Cada um dos três elementos que compõem a dimensão do sistema OKA (PessoaProcesso-Sistema), pode ser utilizado individualmente para medir a Gestão do Conhecimento de uma organização e pode ser capaz de identificar pontos críticos de avaliação destinada à Gestão do Conhecimento.

Por outro lado, o Modelo de Gestão do Conhecimento, elaborado por Fábio Ferreira Batista (2012), visa atender as especificidades e necessidades do setor público. Consiste num modelo hibrido, por contemplar uma sistemática descritiva, destinada a relatar os elementos essenciais da Gestão do Conhecimento e prescritiva, por estabelecer o modus operandi para a implementação da Gestão do Conhecimento. Possui duas partes diretamente interessadas, quais sejam: o usuário, entendido aqui como sujeito individualmente concebido, e a sociedade, vista como uma coletividade determinada ou não (BATISTA, 2012).

Em relação aos cidadãos, usuários do serviço público, a Gestão do Conhecimento volta-se a cumprir a finalidade atribuída à organização pública, ao realizar de forma otimizada os direitos constitucionalmente consagrados em favor dos indivíduos. A referida realização constitui o ponto central de todas as ações desenvolvidas no plano estratégico pela Gestão do Conhecimento junto às organizações públicas (BATISTA, 2012).

O modelo OKA desenvolvido por Fonseca (2006) apresenta-se amplo. Embora seja aplicável à Administração Pública, não foi utilizado para desenvolver a pesquisa, que ora se apresenta, pela exaustividade do instrumento de coleta de dados. Ante a impossibilidade de se dirigir aos 38 (trinta e oito) Institutos Federais de Educação, sediados em todos os Estados brasileiros, adotou-se o Modelo de Gestão do Conhecimento para a Administração Pública Brasileira, desenvolvido por Batista (2012).

Ademais, o Modelo desenvolvido por Batista (2012), por atentar-se às peculiaridades da esfera pública, permite investigar os IFs de forma a enfatizar a parametrização de diversos elementos no ambiente de estudo, bem como ter como referencial analítico os processos

Perspectivas em Gestão \& Conhecimento, João Pessoa, v. 6, n. 2, p. 80-98, jul./dez. 2016 
burocráticos e gerenciais, além da participação dos gestores públicos, em atendimento às peculiaridades do ambiente organizacional público que se apresenta de maneira distinta às exigências que circundam o setor privado.

Enfim, o Modelo de Gestão do Conhecimento para a Gestão Pública Brasileira se insere num contexto social e tecnológico amplo que permite tratar as informações e os conhecimentos gerados no espaço administrativo de forma particularizada, considerando a relevância das ações desenvolvidas no domínio público em prol da coletividade.

\section{TRAJETÓRIA METODOLÓGICA}

Trata-se de pesquisa exploratória e descritiva de caráter misto (GIL, 2002) voltada a aprimorar a compreensão sobre a maturidade na gestão do conhecimento nos IFs.

A pesquisa foi realizada em âmbito nacional, tendo como população os 38 (trinta e oito) IFs, instituídos por intermédio da Lei Federal n.o 11.892/2008, os quais fazem parte da Rede Federal de Educação Profissional, Científica e Tecnológica, que também contempla a Universidade Tecnológica Federal do Paraná - UFTPR, os Centros Federais de Educação Celso Suckow da Fonseca - CEFET/RJ e de Minas Gerais - CEFET/MG, além das escolas técnicas vinculadas às Universidades Federais e o Colégio Pedro II (BRASIL, 2008).

A amostra foi intencional e não probabilística, tendo por parâmetro os setores responsáveis ou relacionados com a Gestão do Conhecimento, em cada uma das instituições integrantes dos IFs. O critério para seleção deu-se mediante a análise dos sites institucionais.

A coleta de dados para a pesquisa foi realizada no segundo semestre de 2014, na qual foram enviados e-mails com questionário para os servidores públicos com atividade funcional vinculada ao tema de estudo. Os e-mails foram direcionados para 28 Pró-Reitores de Desenvolvimento Institucional; quatro Diretores de Tecnologia da Informação; três Próreitores de Administração e Planejamento; um Assessor de Informações e Dados Institucionais; um Diretor de Desenvolvimento Institucional; e um Diretor de Gestão do Conhecimento.

Após a aplicação do questionário, foram obtidas 20 respostas do total de 38 instituições, o que corresponde à adesão de $52,6 \%$ (cinquenta e dois, vírgula seis por cento) das instituições pesquisadas, com respostas integrais, desconsiderando-se as respostas incompletas que inviabilizariam a posterior sistematização e avaliação da maturidade.

Os dados coletados respaldaram-se no questionário eletrônico adaptado do Modelo de Gestão do Conhecimento para Administração Pública (BATISTA, 2012). Na adaptação procedeu-se a inclusão de oito questões preliminares, destinadas a identificar a instituição e o perfil do respondente, como forma de subsidiar as análises qualitativas da maturidade institucional.

A disponibilização e aplicação do questionário deu-se por meio do Programa LimeSurvey, software livre destinado à aplicação de questionários online, o qual permite aos pesquisadores formularem perguntas com a possibilidade de respostas intuitivas. Por tratar-se de um software funcional e autoexplicativo, permite a participação de uma pluralidade de participantes (LIMESURVEY, 2014).

A escolha do modelo desenvolvido por Batista (2012) também se deve à sua especificidade, diretamente voltada à análise da Administração Pública, bem como por sua perspectiva holística, destinada à avaliação integral da Gestão do Conhecimento nas Instituições pesquisadas.

Segundo o modelo adotado, cada assertiva integrante do questionário contempla um peso. A soma de seu conjunto determina o estágio de maturidade, capaz de evidenciar as dimensões da gestão do conhecimento das Instituições Federais de Educação.

Esta sistematização compreende oito categorias. A primeira categoria é composta por oito questões que visam identificar as instituições respondentes e suas atividades de gestão do

Perspectivas em Gestão \& Conhecimento, João Pessoa, v. 6, n. 2, p. 80-98, jul./dez. 2016 
conhecimento. Estas questões subsidiaram as análises das categorias do Modelo de Gestão do Conhecimento para a Administração Pública Brasileira. As demais categorias foram adaptadas do citado modelo e compreendem seis questões, cada qual com cinco alternativas, cujo peso individual variará de um a cinco pontos, a ser atribuído de acordo com a resposta, totalizando 30 pontos cada uma. Com base na pontuação final de cada uma das instituições é possível identificar o seu nível de maturidade na Gestão do Conhecimento.

O Modelo de Gestão do Conhecimento para a Administração Pública Brasileira possui cinco níveis de maturidade, relacionados à pontuação obtida, nos seguintes termos:

1) Reação: de 42 a 83 pontos - consiste no nível em que a organização "não sabe o que é GC e desconhece sua importância para aumentar a eficiência e melhorar a qualidade e a efetividade social" (BATISTA, 2012, p. 95).

II) Iniciação: de 84 a 125 pontos - a instituição "começa a reconhecer a necessidade de gerenciar o conhecimento" (BATISTA, 2012, p. 95).

III) Introdução (expansão): de 126 a 146 pontos - "Há práticas de GC em algumas áreas" (BATISTA, 2012, p. 95).

IV) Refinamento: de 147 a 188 pontos - Neste nível "a implementação da GC é avaliada e melhorada continuamente" (BATISTA, 2012, p. 95).

V) Maturidade: de 189 a 210 pontos. Consiste no nível mais elevado de avaliação da maturidade, onde a Gestão do Conhecimento está institucionalizada na organização objeto de estudo (BATISTA, 2012).

Com base nestes níveis foram analisados os 20 IFs que participaram da pesquisa.

\section{ANÁLISE DOS RESULTADOS}

A análise dos resultados foi realizada pelas categorias do Modelo de Maturidade: aprendizagem e inovação, processo, tecnologia, pessoas, liderança em gestão do conhecimento, processo em gestão do conhecimento, resultado e grau de maturidade da gestão do conhecimento. Dentre os 38 IFS, 20 apresentaram devolutivas e, por questões éticas, não serão identificados pelas suas denominações e sim por regiões, quais sejam: quatro da Região Norte; cinco da Região Nordeste; dois da Região Centro-Oeste; cinco da Região Sudeste, e; quatro da Região Sul.

\subsection{Avaliação do critério de Aprendizagem e Inovação}

O Modelo de Batista (2012), conforme já apresentado, avalia a maturidade da Gestão do Conhecimento com base em sete critérios. A aprendizagem e a inovação contemplam o primeiro critério.

As análises, tendo por parâmetro estes elementos, permitem identificar que o ambiente organizacional está inclinado a articular e reforçar valores relacionados à aprendizagem e à inovação. O erro é considerado uma oportunidade de aprendizagem e desenvolvimento de novas habilidades, com equipes interfuncionais com atribuição para resolver problemas. Os superiores hierárquicos, as chefias intermediárias e os agentes administrativos, por este viés, possuem um perfil mais aberto para a utilização de novas ferramentas e métodos, bem como conferem autonomia para o desenvolvimento das atividades administrativas, com o incentivo pelo trabalho em grupo e pelo compartilhamento das informações (BATISTA, 2012). 
Dessa forma, a percepção individual sobre as experiências e os valores institucionais possibilita a geração do conhecimento organizacional, pois decorre da informação necessária ao processo de aprendizagem individual e coletivo (NONAKA; TAKEUCHI, 1997).

Esta perspectiva confere às organizações o emprego estratégico da informação e lhes permite, com base na aprendizagem, criar, organizar e processar informações tendentes a gerar novos conhecimentos (CHOO, 2006).

Em relação ao objeto de estudo, os resultados isolados relativos ao critério de Aprendizagem e Inovação, em comparação com os níveis de desenvolvimento do Modelo de Gestão do Conhecimento para a Administração Pública Brasileira, foram encontrados os seguintes resultados: i) uma instituição no nível de Maturidade; ii) dez instituições no nível de Refinamento; iii) quatro instituições no nível de Introdução (expansão); iv) quatro instituições no nível de Iniciação, e; v) uma instituição no nível de Reação.

Os resultados de Aprendizagem e Inovação demonstram que os IFs respondentes estão desenvolvendo as atividades relacionadas ao incentivo do trabalho em grupo e ao compartilhamento das informações. Esse critério foi o que apresentou o segundo melhor resultado se comparado aos demais critérios do modelo adotado.

\subsection{Avaliação do critério de Processo}

O processo de Gestão do Conhecimento permite estruturar os objetivos organizacionais e coordenar as metas da instituição. Estas ações são destinadas ao gerenciamento do conhecimento com vistas a auxiliar o aprendizado e a criação de valor da própria organização. (CHOO, 2006).

O critério de processo, no entender de Batista (2012), está atrelado à criação, ao armazenamento, ao compartilhamento e à utilização do conhecimento. Isso decorre de ações sistematizadas, permitindo às organizações disporem e utilizarem um mapa de conhecimento. Nesse caso, há registro e compartilhamento do conhecimento adquirido, pois ele é considerado essencial ao desenvolvimento das atividades institucionais, tanto que é retido quando algum servidor se desvincula da instituição.

A observância dessas características evidencia que as melhores práticas e lições aprendidas são compartilhadas no âmbito organizacional, de modo que é possível a existência de um benchmarking interno e externo voltado não só a melhorar o desempenho, como também a inovar (BATISTA, 2012).

O critério de processo, aplicado aos IFs respondentes, apresenta a terceira melhor média, com 19,45 pontos em relação aos demais critérios. Os resultados isolados deste critério, em comparação com os níveis de desenvolvimento do Modelo de Gestão do Conhecimento para a Administração Pública Brasileira, permitem identificar: i) dez instituições classificadas no nível de Refinamento; ii) seis instituições no nível de Introdução (expansão); iii) três instituições no nível de Iniciação, e; iv) uma instituição no nível de Reação.

\subsection{Avaliação do critério de Tecnologia}

A infraestrutura tecnológica constitui um método eficaz para gerenciar o conhecimento e compartilhar as práticas administrativas (FONSECA, 2006).

Segundo Batista (2012), a avaliação do critério de tecnologia permite identificar o desenvolvimento da gestão do conhecimento que recebe apoio da infraestrutura de tecnologia da informação (TI). Essa área tecnológica tem sua infraestrutura alinhada às estratégias organizacionais e de Gestão do Conhecimento. No plano ideal, caso fosse atingida a pontuação máxima, todos os servidores teriam acesso a computador; à internet e a um endereço de e-mail institucional, cujas informações disponibilizadas pela organização no sítio

Perspectivas em Gestão \& Conhecimento, João Pessoa, v. 6, n. 2, p. 80-98, jul./dez. 2016 
da Internet seriam atualizadas regularmente. A principal fonte de comunicação, transferência e compartilhamento de informações se daria mediante a utilização da Intranet.

Em relação aos resultados isolados do critério de tecnologia e em comparação com os níveis de desenvolvimento do Modelo de Gestão do Conhecimento para a Administração Pública Brasileira, foram encontrados os seguintes resultados: i) cinco instituições no nível de Maturidade; ii) onze instituições no nível de Refinamento; iii) duas instituições no nível de Introdução (expansão), e; iv) duas instituições no nível de Iniciação.

O critério de tecnologia obteve a melhor média em relação aos demais critérios do modelo utilizado. Esta constatação permite inferir que os IFs estão investindo mais nos aspectos tecnológicos.

Conquanto este aporte tecnológico destine-se ao compartilhamento do conhecimento para o desenvolvimento das atribuições funcionais dos servidores públicos que compõem os IFs, um contrassenso identificado consiste na menor importância atribuída pelas instituições ao critério de pessoas, conforme será exposto a seguir.

\subsection{Avaliação do critério de Pessoas}

Os grupos de pessoas estão alocados no âmbito organizacional com o objetivo de resolver problemas e permitir o atingimento dos objetivos institucionais (FONSECA, 2006).

Para Batista (2012), o critério de pessoas está vinculado às diretrizes institucionais. As pessoas compõem o corpo institucional e perseguem os propósitos organizacionais, pautadas em programas educacionais e de capacitação destinados à ampliação do conhecimento e das capacidades dos servidores no desempenho das atividades institucionais. Por este critério, os novos servidores são recebidos numa estrutura disseminadora de informações sobre os benefícios, as políticas, as estratégias, os modelos, os planos e as ferramentas destinados à Gestão do Conhecimento. Com isso, a organização se vale de processos formais de tutoria, mentoring e coaching, além de um banco de competências, atrelado ao reconhecimento e à recompensa dos servidores colaboradores que compartilham o conhecimento, primando pelo trabalho em equipe.

Em relação aos resultados isolados do critério de Pessoas, em comparação com os níveis de desenvolvimento do Modelo de Gestão do Conhecimento para a Administração Pública Brasileira, foi encontrada a seguinte composição: i) sete instituições no nível de Introdução (expansão); ii) 10 instituições no nível de Iniciação, e; iii) três instituições no nível de Reação.

O critério de Pessoas ocupa a quinta média, em relação aos demais critérios, atingindo 15,3 pontos, superando apenas os critérios de Processos de Gestão do Conhecimento e de Resultados de Gestão de Conhecimento.

Este resultado mostra-se incongruente, pois o critério de pessoas constitui o elemento crucial para a Gestão do Conhecimento nas organizações.

\subsection{Avaliação do critério de Liderança em Gestão do Conhecimento}

Os gestores, no exercício de suas atribuições, podem desenvolver uma visão holística do conhecimento, pois no exercício da liderança desenvolvem processos criativos tendentes ao compartilhamento do conhecimento (TAKEUCHI; NONAKA, 2008).

Para Batista (2012), a avaliação do critério de liderança em gestão do conhecimento tem por escopo o alinhamento da gestão e da estratégia de Gestão do Conhecimento com os demais direcionadores estratégicos organizacionais e seus possíveis compartilhamentos; a disponibilidade de arranjos organizacionais; a alocação de recursos financeiros; a existência de política destinada à proteção do conhecimento; a existência de trabalho colaborativo e de

Perspectivas em Gestão \& Conhecimento, João Pessoa, v. 6, n. 2, p. 80-98, jul./dez. 2016 
compartilhamento do conhecimento entre a alta administração e as chefias intermediárias enquanto modelo a ser seguido pelos demais servidores; a existência de estímulo pela alta administração e pelas chefias intermediárias aos servidores que seguem seus métodos de trabalhos, estando todos estes elementos relacionados às iniciativas organizacionais de gestão do conhecimento.

Em relação aos resultados isolados no critério de Liderança em Gestão do Conhecimento, em comparação com os níveis de desenvolvimento do Modelo de Gestão do Conhecimento para a Administração Pública Brasileira, há a seguinte constatação: i) uma instituição no nível de Maturidade; ii) duas instituições no nível de Refinamento; iii) cinco instituições no nível de Introdução (expansão); iv) nove instituições no nível de Iniciação, e; v) três no nível de Reação. A média encontrada para critério é de 16 pontos, ocupando a quarta posição em relação às médias dos demais critérios.

\subsection{Avaliação do critério de Processo de Gestão do Conhecimento}

A produção de novos conhecimentos exige a adoção de processos sistêmicos, bem como a interação entre os conhecimentos tático e explícito (TAKEUCHI; NONAKA, 2008).

O preenchimento do critério de processos de Gestão do Conhecimento, para Batista (2012) exige o atendimento dos seguintes requisitos:

i) a organização tem processos sistemáticos de identificação, criação, armazenamento, compartilhamento e utilização do conhecimento; ii) a organização conta com um mapa de conhecimento e o utiliza; iii) o conhecimento adquirido é registrado e compartilhado; iv) o conhecimento essencial dos servidores que estão saindo da organização é retido; v) se a organização compartilha as melhores práticas e lições aprendidas; e vi) se há benchmarking interno e externo para melhorar o desempenho e inovar (BATISTA, 2012, p. 92)

Conforme o entendimento apresentado, a dinâmica organizacional reflete o processo de gestão do conhecimento adotado. Na prática, os resultados atribuídos ao critério de processo de Gestão do Conhecimento nos IFs, em comparação com os níveis de desenvolvimento do Modelo de Gestão do Conhecimento para a Administração Pública Brasileira, observaram-se: i) uma instituição no nível de Maturidade; ii) cinco instituições no nível de Introdução (expansão); iii) seis instituições no nível de Iniciação, e; iv) oito instituições no nível de Reação. A média deste critério ficou 14,05 pontos, sendo a segunda pior média em relação aos demais critérios adotados pelo modelo utilizado.

\subsection{Avaliação do critério de Resultado da Gestão do Conhecimento}

O critério referente ao "Resultado da Gestão do Conhecimento" está ligado ao histórico de implementação da gestão do conhecimento no âmbito organizacional, além da utilização dos indicadores de avaliação de impacto sobre as contribuições e as iniciativas da gestão do conhecimento. Neste ambiente, a Gestão do Conhecimento contribui para a busca de melhoria nos resultados relativos aos indicadores de eficiência e àqueles vinculados à qualidade; à efetividade social e aos princípios da legalidade, da impessoalidade, da publicidade e da moralidade administrativa (BATISTA, 2012).

Em relação aos resultados isolados do critério de Resultados de Gestão do Conhecimento, em comparação com os níveis de desenvolvimento do Modelo de Gestão do Conhecimento para a Administração Pública Brasileira, podem ser destacadas as seguintes

Perspectivas em Gestão \& Conhecimento, João Pessoa, v. 6, n. 2, p. 80-98, jul./dez. 2016 
classificações: i) uma instituição no nível de Refinamento; ii) uma instituição no nível de Iniciação, e; iii) 18 instituições no nível de Reação. Este critério obteve a média de 6,25 pontos, ocupando a pior posição dentre as médias encontradas para os demais critérios do modelo utilizado.

\subsection{Grau de Maturidade da Gestão do Conhecimento no IFs}

Conforme referenciado anteriormente, o grau de maturidade da Gestão do Conhecimento deve ser analisado com base nos critérios de Aprendizagem e Inovação; Processo; Tecnologia; Pessoas; Liderança em Gestão do Conhecimento; Processo de Gestão do Conhecimento, e; Resultado da Gestão do Conhecimento.

Todos os critérios referenciados foram individualmente aplicados aos IFs. O Quadro 2 apresenta o comparativo relativo ao maior grau, o menor grau e o grau médio de maturidade obtido pelas instituições analisadas.

A relevância deste Quadro decorre da possibilidade de, por meio dos valores nele referenciados, verificar o grau médio da maturidade na Gestão do Conhecimento dos IFs, bem como compará-los com os valores encontrados na rede, principalmente quanto ao maior grau e o menor grau de maturidade encontrado nas instituições.

Quadro 2 - Comparativo entre o maior grau, o menor grau e o grau médio de maturidade

\begin{tabular}{|l|r|r|r|r|r|r|r|}
\hline Maturidade & $\begin{array}{c}\text { Aprendizagem e } \\
\text { Inovação }\end{array}$ & Processo & Tecnologia & Pessoas & $\begin{array}{c}\text { Liderança } \\
\text { em GC }\end{array}$ & $\begin{array}{c}\text { Processo de } \\
\text { GC }\end{array}$ & $\begin{array}{c}\text { Resultado } \\
\text { da GC }\end{array}$ \\
\hline $\begin{array}{l}\text { Maior Grau } \\
\text { de } \\
\text { Maturidade }\end{array}$ & 24,00 & 24,00 & 27,00 & 20,00 & 14,00 \\
\hline $\begin{array}{l}\text { Menor Grau } \\
\text { de } \\
\text { Maturidade }\end{array}$ & 9,00 & 11,00 & 19,00 & 8,00 & 10,00 & 8,00 & 6,00 \\
\hline $\begin{array}{l}\text { Grau Médio } \\
\text { de } \\
\text { Maturidade }\end{array}$ & 20,60 & 19,45 & 23,55 & 15,30 & 16,00 & 14,05 & 6,25 \\
\hline
\end{tabular}

Fonte: Dados da pesquisa (2015)

As instituições em destaque focaram seus investimentos em tecnologia. Porém, o critério de pessoas ficou abaixo dos critérios de aprendizagem e inovação, processos, tecnologia e liderança em Gestão do Conhecimento, tanto no que tange ao grau médio considerado a ponderação de todos os valores identificados nas instituições pesquisadas em relação a cada critério isolado -, quanto ao maior e ao menor grau de maturidade.

A realização destas inferências também pode ser interpretada por meio do Quadro 3 que apresenta o nível de maturidade em Gestão do Conhecimento relativa a todos os institutos pesquisados.

Quadro 3- Nível de maturidade em Gestão do Conhecimento

\begin{tabular}{|l|c|c|}
\hline \multicolumn{1}{|c|}{ Instituição } & $\begin{array}{c}\text { Grau de } \\
\text { Maturidade }\end{array}$ & $\begin{array}{c}\text { Nível de Maturidade em Gestão do } \\
\text { Conhecimento }\end{array}$ \\
\hline Instituto Federal 01 & 151 & Refinamento \\
\hline Instituto Federal 02 & 148 & Refinamento \\
\hline Instituto Federal 03 & 133 & Introdução (expansão) \\
\hline Instituto Federal 04 & 131 & Introdução (expansão) \\
\hline Instituto Federal 05 & 130 & Introdução (expansão) \\
\hline Instituto Federal 06 & 129 & Introdução (expansão) \\
\hline
\end{tabular}

Perspectivas em Gestão \& Conhecimento, João Pessoa, v. 6, n. 2, p. 80-98, jul./dez. 2016 


\begin{tabular}{|l|c|c|}
\hline Instituto Federal 07 & 126 & Introdução (expansão) \\
\hline Instituto Federal 08 & 123 & Iniciação \\
\hline Instituto Federal 09 & 123 & Iniciação \\
\hline Instituto Federal 10 & 120 & Iniciação \\
\hline Instituto Federal 11 & 113 & Iniciação \\
\hline Instituto Federal 12 & 111 & Iniciação \\
\hline Instituto Federal 13 & 110 & Iniciação \\
\hline Instituto Federal 14 & 109 & Iniciação \\
\hline Instituto Federal 15 & 109 & Iniciação \\
\hline Instituto Federal 16 & 98 & Iniciação \\
\hline Instituto Federal 17 & 94 & Iniciação \\
\hline Instituto Federal 18 & 93 & Iniciação \\
\hline Instituto Federal 19 & 82 & Reação \\
\hline Instituto Federal 20 & $\mathbf{7 1}$ & Reação \\
\hline Nível Médio de Grau de Maturidade & $\mathbf{1 1 5 , 2}$ & Iniciação \\
\hline
\end{tabular}

Fonte: Dados da pesquisa (2015)

O Quadro 3 demonstra, a partir dos pontos atribuídos ao conjunto dos critérios respondidos por cada instituição, o nível de maturidade em Gestão do Conhecimento no âmbito dos IFs, qual seja: duas instituições no nível de reação; onze instituições no nível de iniciação; cinco instituições no nível de introdução (expansão), e; duas instituições no nível de refinamento. O nível médio do grau de maturidade demonstra que os IFs, como um todo, encontram-se no nível de iniciação.

Para Batista (2012), as organizações, no nível de iniciação, considerando o contexto institucional, estão tomando ciência das reais necessidades de gerenciar o conhecimento para atingirem os objetivos aos quais se propõem a desenvolver. Ainda significa a ausência de práticas ou de implementação de Gestão do Conhecimento em algumas áreas, bem como de melhorias contínuas neste âmbito, não estando institucionalizada a Gestão do Conhecimento nas organizações públicas.

O estudo apresentado permite concluir, especialmente pela existência de apenas duas instituições no nível de maturidade de refinamento, que a Gestão do Conhecimento é incipiente nos IFs. Na prática, a maioria dos respondentes ainda não reconheceu a importância da Gestão do Conhecimento enquanto método capaz de permitir o desenvolvimento institucional no cumprimento de seus objetivos.

\section{CONSIDERAÇÕES FINAIS}

As organizações públicas ou privadas se valem da Gestão do Conhecimento como instrumento hábil ao desempenho das funções organizacionais de âmbito interno ou externo. Todavia, a ampla disponibilização de informações exige a adoção de sistemas de controle destinados a atingir os objetivos organizacionais.

É nesse contexto que se inserem os modelos de maturidade, com competência para avaliar se as ações de Gestão do Conhecimento estão alinhadas com os objetivos organizacionais. Por derivarem de uma visão sistêmica, permitem identificar e orientar estrategicamente os agentes na implementação das ações, no planejamento e controle de seus resultados.

A aplicação do Modelo de Gestão do Conhecimento para a Administração Pública Brasileira, desenvolvido por Batista (2012) para a análise dos IFs, que compõem a Rede Federal de Educação, permite destacar algumas evidências. 
Os IFs são estabelecidos em âmbito nacional e têm suas estruturas pautadas num critério legislativo comum (Lei n.o 11.892/2008). Porém, a administração realizada, individualmente pelos órgãos que os compõem, mostra-se heterogênea. Ou seja, não há uma simetria quanto ao modelo de gestão adotado pelos IFs. Consequentemente, por mais que se reconheça a relevância atribuída à Gestão do Conhecimento, a aplicação de suas premissas e orientações mostra-se discutível. Neste caso, os dados obtidos permitem concluir, inclusive, pelo desconhecimento do assunto por grande parte das instituições.

Estes fatores compõem os resultados obtidos com a análise individual de todos os critérios integradores do Modelo de Gestão do Conhecimento para a Administração Pública Brasileira. Portanto, é possível inferir as dificuldades encontradas pelos IFs na realização dos seus princípios institucionais, especialmente aqueles atrelados à eficácia e eficiência da gestão da coisa pública.

O ápice da maturidade buscada pela Gestão do Conhecimento exige a participação ativa dos agentes, com disposição para alterar os hábitos individuais e as rotinas organizacionais, no intuito de atingir o sucesso institucional.

No âmbito da administração pública, a proatividade organizacional destinada à alocação sistemática das informações, constitui elemento indispensável à tomada de decisão e à realização dos objetivos institucionais. Todavia, a realidade teórica destoa da prática, uma vez que o ambiente estudado (IFs) encontra-se inaugurando suas ações destinadas a gerir o conhecimento organizacional, porquanto a maioria das instituições contempla o grau de maturidade de iniciação.

A análise apresentada aponta pela necessidade de aprofundamento nos estudos sobre a Gestão do Conhecimento no âmbito dos IFs, destinado ao acompanhamento dos indicadores de gestão e da mensuração dos níveis de maturidade na Gestão do Conhecimento por um período suficiente para ser possível o estabelecimento de uma correlação entre os resultados dos indicadores de gestão e os níveis de maturidade do modelo adotado.

Por ora, conclui-se pela perenidade na sistematização e organização da gestão do conhecimento pelos IFs. Estas instituições de ensino se encontram inseridas num processo de desafios e conflitos constantes na busca por uma forma gerencial apta a atender às peculiaridades deste setor público de ensino. A resistência e a persistência de modelos burocráticos e gerenciais inadequados para a atual conjuntura administrativa do Estado apontam pela necessidade desta transitoriedade para um novo Modelo de Gestão do Conhecimento.

\section{REFERÊNCIAS}

ABDULLAH, K; DATE, H. Public sector knowledge management: a generic framework. Public Sector ICT Management Review, v. 3, n. 1. 2009. Disponível em: http://www.researchgate.net/publication/265864864 PUBLIC SECTOR KNOWLEDGEMANAG EMENT A GENERIC FRAMEWORK. Acesso em: 12 set. 2014.

AHMED, P. K.; LIM, K. K.; ZAIRI, M. Measurement practice for knowledge management. Journal of Workplace Learning, v. 11, n. 8, p. 304-311, 1999. Disponível em: http://dx.doi.org/10.1108/13665629910300478. Acesso em: 04 nov. 2014.

ALATAWI, F.M.H; DWIVEDI, Y.K.; WILLIAMS, M.D. A Review of Knowledge Management Research in Public Sector Context with a Specific Focus on Arab Countries. International Journal of Business Information Systems, v.14, n.1, p.56-82, 2013.

ALVARENGA NETO, R. C. D. Gestão do conhecimento em organizações: proposta de mapeamento conceitual integrativo. São Paulo: Saraiva, 2008. 
ANGELONI, Maria Terezinha; MUSSI, Clarissa Carneiro. Compartilhamento doConhecimento no Contexto de Projetos de Tecnologia da Informação. In: ANGELONI, M. T. (Org). Gestão do conhecimento no Brasil: casos, experiências e práticas de empresas públicas. Rio de Janeiro: Qualitynark, 2008.

BARRADAS, J. S.; CAMPOS FILHO, L. A. N. Levantamento de tendências em gestão do conhecimento no Brasil: análise de conteúdo da opinião de especialistas brasileiros. Perspectivas em Ciência da Informação, v. 15, n. 3, p. 131-154, set/dez, 2010. Disponível em: http://www.scielo.br/pdf/pci/v15n3/08.pdf. Acesso em: 04 nov. 2014.

BATISTA, F. F. Modelo de gestão do conhecimento para a administração pública brasileira: como implementar a gestão do conhecimento para produzir resultados em benefício do cidadão. Brasília: IPEA, 2012. Disponível em: http://repositorio.ipea.gov.br/handle/11058/754. Acesso em: 04 nov. 2014.

BOURGON, J. Review of Administrative Sciences. International Review of Administrative Sciences, v. 73 , p. 1, 2007. Disponível em: http://web.iaincirebon.ac.id/ ebook/moon/Bureaucracy-Governance/Responsive,\%20responsible\%20and\%20respected\%20 government.pdf. Acesso em 12 set. 2014.

BRASIL. Lei n. 11.892, de 29 de dezembro de 2008. Institui a Rede Federal de Educação Profissional, Científica e Tecnológica, cria os Institutos Federais de Educação, Ciência e Tecnologia, e dá outras providências. Diário Oficial da República Federativa do Brasil, Brasília, DF, n. 253, p. 1, 30dez. 2008. Seção 1, p. 1. Disponível em: http://pesquisa.in.gov.br/imprensa/isp/visualiza/index.jsp?jornal=1\&pagina=1\&data=30/12/2 008. Acesso em: 04 ago. 2014.

CARVALHO, I. M. Estratégias para implantação de Gestão do Conhecimento. In: ANGELONI, M. T. (Org). Gestão do conhecimento no Brasil: casos, experiências e práticas de empresas públicas. Rio de Janeiro: Qualitynark, 2008.

CHOO, C. W. A organização do conhecimento: como as organizações usam a informação para criar significado, construir conhecimento e toma decisões. 2. ed. São Paulo: Editora Senac, 2006.

DALE, A. Dispatches: letters from the corporanian war zone. Journal of Information Science, aug., 2001.

DENHARDT, R. B; DENHARDT, J. V. The New Public Service: Serving Rather Than Steering. Public Administration Review, v. 60, n. 6, p. 549-559, 2000. Disponível em: http://www.jstor.org/stable/977437. Acesso em 12 set. 2014.

EDGE, $K$. The power of public sector knowledge management: The case of an education district making strides. Journal of Knowledge Management. v. 6, n. 9, p. 42-52, 2005.

FIATES, G.G.S. Cultura Organizacional: Um fator Determinante para a Promoção da Aprendizagem Organizacional e da Gestão do Conhecimento. In: ANGELONI, M. T. (Org). Gestão do conhecimento no Brasil: casos, experiências e práticas de empresas públicas. Rio de Janeiro: Qualitynark, 2008.

FONSECA, A. F. Organizational Knowledge Assessment Methodology. Washington, DC: World Bank, 2006. Disponível em: http://www.nti.ufpb.br/ evandro/oka/2006 0611 v6 OKA Completion Repor t\%20( 2).pdf. Acesso em 09 nov. 2014. 
FRESNEDA, P. S. V. et al. Diagnóstico da gestão do conhecimento nas organizações públicas utilizando o método organizacional KnowledgeAssessment (OKA). 2009. Disponível em: http://www.repositorio.

fjp.mg.gov.br/consad/bitstream/123456789/268/1/C2 TP DIAGN\%C3\%93STICO\%20DA\%20GE ST\%C3\%830\%20DO\%20CONHECIMENTO.pdf. Acesso em 09 nov. 2014.

FULLAN, M. Leading in a Culture of Change: Being Effective in Complex Times. San Francisco: Jossey-Bass, 2001.

GIL, A. C. Como elaborar projetos de pesquisa. 4. ed. São Paulo: Atlas, 2002.

GONÇALO, C. R.; JUNGES, F. M.; BORGES, M. L. Avaliação da gestão do conhecimento: modelos de mensuração. ENCONTRO NACIONAL DE ENGENHARIA DE PRODUÇÃO, 30., São Carlos. Anais... São Carlos, SP, Brasil, 12 a 15 de outubro de 2010. Disponível em: http://projeto.unisinos.br/gp gestaoconhecimento/sites/default/files/publicacoes/2010/TN S TO 120784 17312.pdf. Acesso em: 10 nov. 2013.

GOULART, S. Gerenciando Sistemas de Conhecimento. In: ANGELONI, M. T. (Org). Gestão do conhecimento no Brasil: casos, experiências e práticas de empresas públicas. Rio de Janeiro: Qualitynark, 2008.

HAGGIE, K.; KINGSTON, J. Choosing your knowledge management strategy. Journal of Knowledge Management Practice, jun., 2003.

JAIN, Priti. Knowledge Management in E-Government. Journal of Knowledge Management Practice, v. 10, n. 4, 2009.

LIMESURVEY. LimeSurvey Manual. Disponível em: http://manual.limesurvey.org/ LimeSurvey Manual. Acesso em 05 ago. 2014.

LING, T. Delivering joined-up government in the UK: dimensions, issues and problems. Public Administration, v. 80, n. 4, p.615-642, winter, 2002.

LUEN, T.; AL-HAWAMDEH, S. Knowledge management in the public sector: principles and Management. Journal of information Science, v. 7, n. 4, p. 303-313, 2001.

MARICATO, E. et al. Cidades rebeldes: passe livre e as manifestações que tomaram as ruas do Brasil. São Paulo: Boitempo/Carta Maior, 2013.

MCADAM, R.; REID, R. A comparison of public and private sector perceptions and use of knowledge management. Journal of European Industrial Training, v. 24 n. 6, p. 317. 2001. Disponível em: http://dx.doi.org/10.1108/03090590010346424. Acesso em 12 set. 2014.

NONAKA, I.; TAKEUCHI, H. Criação de conhecimento na empresa: como as empresas japonesas geram a dinâmica da inovação. Tradução: Ana Beatriz Rodrigues; Priscilla Martins Celeste. 14. ed. Rio de Janeiro: Campus, 1997.

OLIVEIRA, M. et al. Proposta de um modelo de maturidade para Gestão do Conhecimento: $\mathrm{KM}^{3}$. Revista Portuguesa e Brasileira de Gestão, Lisboa, v. 10, n.4, out.2011. Disponível em http://www.scielo.gpeari.mctes.pt/scielo.php?script=sci arttext\&pid=S1645-446420110003 00003\&lng=pt\&nrm=iso. Acesso em: 04 nov. 2013.

OLIVEIRA, R. R; TORRES, M. F. P. Inovação e Pesquisa \& Desenvolvimento no Setor Eletrico Chesf: Uma Empresa na Era do Conhecimento. In: ANGELONI, M. T. (Org). Gestão do conhecimento no Brasil: casos, experiências e práticas de empresas públicas. Rio de Janeiro: Qualitynark, 2008. 
PEREIRA, L. A. C. Institutos Federais de Educação Ciência e Tecnologia. 2008. Disponível em: http://www.educacao.gov.br/setec/arquivos/pdf3/artigos ifet jornal.pdf. Acesso em: 10 jul., 2014.

RAMANIGOPAL, C. Knowledge Management Strategies for Successful Implementation in Aerospace Industry. Advances in Management, v. 5, p. 12, 2012. Disponível em: http://www.managein.net/pdf Articles/December 2012/17-21=Article\%203.pdf. Acesso em: 11 jul., 2013.

SCHLESINGER, C. C. B., et al.Gestão do Conhecimento na Administração Pública. Curitiba: Instituto Municipal de Administração Pública - IMAP, 2008.

SILVA, H.F.N. O processo de Criação de Conhecimento Organizacional em Comunidades de Prática. In: ANGELONI, M. T. (Org). Gestão do conhecimento no Brasil: casos, experiências e práticas de empresas públicas. Rio de Janeiro: Qualitynark, 2008.

TAKEUCHI, H; NONAKA, I. Gestão do conhecimento. Porto Alegre: Bookman. 2008.

TERRA, J. C. C. Gestão do conhecimento: o grande desafio empresarial. TerraForum, 2005. Disponível em: http://biblioteca.terraforum.com.br/Paginas/OGrandeDesafioEmpresarial !.aspx. Acesso em: 10 jul. 2013.

VAN BEVEREN, J. Does health care for knowledge management? Journal of Knowledge Management, v. 6, n. 5, p. 420-33, 2003.

WIIG, K.M. Knowledge management in public administration. Journal of Knowledge Management, $\quad$ v. $6 \quad$ n. 3 , p. 224-39, 2002. Disponível em: http://dx.doi.org/10.1108/13673270210434331. Acesso em: 14 set. 2014.

Artigo recebido em 19/11/2015 e aceito para publicação em 09/05/2016 